\title{
Characteristics of elastic anisotropy of Ti-Ni crystals with shape memory and their relation to extreme values of the Poisson's ratio
}

\author{
S. A. Muslov \\ muslov@mail.ru
}

\author{
A. I. Yevdokimov Moscow State University of Medicine and Dentistry, Moscow, 127473, Russia
}

\begin{abstract}
The study of physical and mechanical properties can shed light on the picture of martensitic transitions in materials that control the unique effects of shape memory and superelasticity. Martensitic transformations in metals and alloys are based on shear-type deformation processes, so the study of their elastic behavior contains important information about the nature and mechanisms of these phase transitions. In this report, we analyze the value and relationship between the degree of elastic anisotropy and the Poisson's ratio of Ti-Ni alloy crystals based on our own and literature data obtained experimentally and computationally from the first principles. A significant variability of the elastic anisotropy coefficient $A$ of Ti-Ni crystals was established, which is due to the apparently elastic "softening" of the alloy lattice on the eve of martensitic transformations (especially the constant c44). The minimum Poisson's ratio of Ti-Ni crystals is -0.25 . The maximum value of $\mu$ was 1.10 , which is higher than the theoretical limit for polycrystals. Extreme values of $\mu$ were achieved for directions when the stretch is oriented along the direction $<110>$, i. e. diagonals of the cube face. The mean $<\mu>$ was found to be 0.41 . Ti-Ni based alloys can be considered as partial auxetic — materials with negative Poisson's ratio in some directions, that is, have $\mu<0$ when choosing some directions and be an ordinary material with positive $\mu$ for other directions. The elastic anisotropy of Ti-Ni crystals can be determined based on the numerical differences in the extreme values of $\mu$ as $A_{\mu}=\left|\mu_{\max }-\mu_{\min }\right| /\langle\mu>$. A strong correlation was established between the elastic anisotropy coefficient $A$ and the factor $A_{\mu}$ (the correlation coefficient 0.9871 ). The parameters of the linear dependence of the reduced anisotropy factor $A_{\mu}{ }^{*}=1.33779 A_{\mu}+0.18542$, for which $A_{\mu}^{*}=A$.
\end{abstract}

Keywords: elastic anisotropy, Poisson's ratio, Ti-Ni.

УДК: 669

\section{Характеристики упругой анизотропии кристаллов $\mathrm{Ti}-\mathrm{Ni}$ c памятью формы и их связь с экстремальными значениями коэффициента Пуассона}

\author{
Муслов С. А. \\ Московский государственный медико-стоматологический университет (МГМСУ) им. А. И. Евдокимова, \\ Москва, 127473, Россия
}

Исследование физико-механических свойств способно пролить свет на картину мартенситных переходов в материалах, ответственных за уникальные эффекты памяти формы и сверхэластичности. В основе мартенситных превращений в металлах и сплавах лежат деформационные процессы сдвигового типа, поэтому изучение их упругого поведения содержит важную информацию о природе и механизмах этих фазовых переходов. В данном сообщении анализируется величина и связь степени упругой анизотропии и коэффициента Пуассона кристаллов сплавов Тi-Ni на основе собственных и литературных данных, полученных опытным и расчетным путем из первых принципов. Установлена значительная вариабельность коэффициента упругой анизотропии $A$ кристаллов Ti-Ni, обусловленная, очевидно, интенсивным упругим «размягчением» решетки сплавов накануне мартенситных превращений (особенно постоянной с44). Минимальный коэффициент Пуассона кристаллов Ti-Ni равен -0.25. Максимальное значение $\mu$ составило 1.10, что больше теоретического предела для поликристаллов. Экстремальные значения $\mu$ достигались для направлений, когда растяжение было ориентировано вдоль направления <110>, то есть диагоналей грани куба. Интегрированием по поперечным направлениям и направлениям приложения силы установлено, что среднее значение $<\mu>0.41$. Сплавы на основе Ti-Ni могут быть рассмотрены, таким образом, как частичные ауксетики материалы с отрицательным коэффициентом Пуассона в части направлений, то есть иметь $\mu<0$ при выборе одних направлений и быть обыкновенными материалами с положительным $\mu$ для других направлений. Упругая 
анизотропия кристаллов Ti-Ni может быть определена на основе численных различий экстремальных значений $\mu$ как $A_{\mu}=\left|\mu_{\max }-\mu_{\min }\right| /<\mu>$. Установлена сильная корреляционная связь между коэффициентом упругой анизотропии $A$ и фактором $A_{\mu}$ (коэффициент корреляции 0.9871). Эмпирически установлены параметры линейной зависимости приведенного фактора анизотропии $A_{\mu}^{*}=1.33779 A_{\mu}+0.18542$, для которого $A_{\mu}^{*}=A$.

Ключевые слова: упругая анизотропия, коэффициент Пуассона, Ti-Ni.

\section{1. Введение}

Данные об упругих постоянных $c_{i j}$ монокристаллов непосредственно отражают характер и величину межатомных связей, а также устойчивость исходной фаз и формированию предмартенситных и мартенситных структур. В линейном приближении упругие свойства кубических кристаллов полностью описываются матрицей

$$
\left|\begin{array}{cccccc}
c_{11} & c_{12} & c_{12} & 0 & 0 & 0 \\
c_{12} & c_{11} & c_{12} & 0 & 0 & 0 \\
c_{12} & c_{12} & c_{11} & 0 & 0 & 0 \\
0 & 0 & 0 & c_{44} & 0 & 0 \\
0 & 0 & 0 & 0 & c_{44} & 0 \\
0 & 0 & 0 & 0 & 0 & c_{44}
\end{array}\right|,
$$

которая содержит три независимые упругие постоянные $c_{11}, c_{12}$ и $c_{44}$. Из них только постоянная $c_{44}$ имеет прямой физический смысл как мера сопротивления кристалла сдвигу в плоскости куба [100] вдоль любого направления $<0 \mathrm{kl}>$ в этой плоскости. Коэффициенты матрицы $c_{11}$ и $c_{12}$ такого простого объяснения не имеют. Однако их линейные комбинации $B=\left(c_{11}+2 c_{12}\right) / 3$ и $C^{\prime}=\left(c_{11}-c_{12}\right) / 2$ являются, в первом случае, мерой сопротивления кристаллов гидростатическому сжатию (модуль объемной упругости) и, во втором случае, мерой сопротивления кристалла сдвигу плоскости $\{110\}$ в направлениях $<1 \overline{1} 0>$. При этом, $c_{44}$ и $C^{\prime}-$ экстремальные (наибольший и наименьший) модули сдвига в кубическом кристалле (реже наоборот). Степень упругой анизотропии кубических кристаллов обычно аттестуется безразмерным отношением С. Zener, 1948 [1]

$$
A=c_{44} / C^{\prime}=2 c_{44} /\left(c_{11}-c_{12}\right) .
$$

Оно характеризует относительную степень сопротивления кристаллов кубической сингонии двум основным типам сдвиговой деформации в них. Очевидно, что для упруго-изотропного кристалла $A=1, c_{44}=C^{\prime}$, $2 c_{44}=c_{11}-c_{12}$. Металлы и сплавы упруго анизотропны и как правило $A>1$. Степень упругой анизотропии металлов и сплавов с мартенситными превращениями в высокотемпературной фазе (аустенитном состоянии) весьма вариабельна (Рис. S1, дополнительный материал). При этом в предмартенситном состоянии отдельных интерметаллидов она может дополнительно изменяться вследствие интенсивного "размягчения» части постоянных и их комбинаций [2]. Как правило образующиеся «мягкие» длинноволновые моды соответствуют вариантам кристаллографической перестройки решетки при фазовых переходах.

Коэффициент Пуассона отражает особенности поперечной деформации, происходящей в направлении, не совпадающем с направлением действия внешней силы. Поперечная деформация определяется свойством тела передавать внешнее воздействие в других направлениях, что зависит от атомно-молекулярного строения тела и динамики решетки. Коэффициент Пуассона характеризует стремление материала сохранить свой объем при упругой деформации и для произвольного направления представляет собой отношение поперечного сокращения к продольному удлинению при растяжении материала, взятое с обратным знаком $\mu=\varepsilon_{\perp} / \varepsilon_{\|}$. Для упругих тел, увеличивающих свой объем при растяжении и уменьшающих при сжатии, величина $\mu$ лежит в пределах от 0 до 0.5. Для большинства материалов практически диапазон коэффициента Пуассона ещё уже: от 0.25 до 0.35. Однако по современным представлениям интервал возможных значений коэффициента Пуассона может быть существенно расширен за оба предела: $\mu$ может быть и отрицательным $(\mu<0)$ и больше 0.5 [3]. Хотя термодинамика не запрещает отрицательное отношение Пуассона (то есть боковое расширение в ответ на растяжение), это свойство обычно считается редким в кристаллических твердых телах. В противоположность этому убеждению, 69\% кубических элементарных металлов имеют отрицательное отношение Пуассона при растяжении вдоль направления [110] [4]. Отрицательные коэффициенты Пуассона также наблюдались в ряде материалах вблизи фазовых переходов [5].

Цель работы: на основе собственных и литературных данных определить значения коэффициента Пуассона кристаллов сплавов $\mathrm{Ti}-\mathrm{Ni}$ с мартенситными превращениями и памятью формы, их пространственную вариабельность и возможность использования изменчивости $\mu$ при оценке упругой анизотропии кристаллов.

\section{2. Методы расчета}

Степень упругой анизотропии кристаллов оценивали по формуле (1).

Экстремальные значения коэффициента Пуассона рассчитывали по формулам для кубических кристаллов (Wang X. F. et al., 2012) [6]:

$$
\begin{aligned}
& \mu_{\min }=\mu([110],[1 \overline{10}])=-\frac{2 c_{11} c_{44}-\left(c_{11}-c_{12}\right)\left(c_{11}+2 c_{12}\right)}{2 c_{11} c_{44}+\left(c_{11}-c_{12}\right)\left(c_{11}+2 c_{12}\right)}, \\
& \mu_{\max }=\mu([110],[001])=\frac{4 c_{12} c_{44}}{2 c_{11} c_{44}+\left(c_{11}-c_{12}\right)\left(c_{11}+2 c_{12}\right)} .
\end{aligned}
$$

Как видно экстремальные значения $\mu$ достигаются для направлений, когда растяжение ориентировано вдоль направления $<110>$, то есть диагонали грани куба.

В общем случае коэффициент Пуассона является функцией трех углов $\mu=\mu(\varphi, \theta, \alpha)$, два из которых $\varphi$, 
$\theta$ показывают направление продольной силы и один $\alpha$ - направление, перпендикулярное продольной силе [7]. Усреднением по всем поперечным направлениям $\alpha$ получаем $\mu_{\mathrm{p}}=\mu_{\mathrm{p}}(\varphi, \theta)=\int \mu(\varphi, \theta, \alpha) / \int \mathrm{d} \alpha$. Средний коэффициент Пуассона $<\mu>$ вычисляли как определенный интеграл

$$
<\mu>=\frac{1}{2 \pi} \int_{0}^{\pi} \mathrm{d} \varphi \int_{0}^{\pi} \sin \theta \mu_{\mathrm{p}}(\varphi, \theta) \mathrm{d} \theta,
$$

где $\mu_{\mathrm{p}}(\varphi, \theta)$ - коэффициент Пуассона как функция от 2-х углов, усредненная по поперечным направлениям,

$$
\mu_{\mathrm{p}}(\varphi, \theta)=-\frac{a r_{12}+b\left(r_{44}-2\right)}{16\left[c+d\left(2 r_{12}+r_{44}\right)\right]},
$$

где

$$
\begin{gathered}
a=2\left(53+4 \cos 2 \theta+7 \cos 4 \theta+8 \cos 4 \varphi \sin ^{4} \theta\right), \\
b=-11+4 \cos 2 \theta+7 \cos 4 \theta+8 \cos 4 \varphi \sin ^{4} \theta, \\
c=9 \cos ^{4} \theta+6 \sin ^{4} \theta+2 \cos 4 \varphi \sin ^{4} \theta \\
d=2\left(\sin ^{2} 2 \theta+\sin ^{4} \theta \sin ^{2} 2 \varphi\right), \\
r_{12}=\frac{s_{12}}{s_{11}}, r_{44}=\frac{s_{44}}{s_{11}}, \\
s_{11}=\frac{c_{11}+c_{12}}{\left(c_{11}-c_{12}\right)\left(c_{11}+2 c_{12}\right)}, s_{12}=\frac{-c_{12}}{\left(c_{11}-c_{12}\right)\left(c_{11}+2 c_{12}\right)}, s_{44}=\frac{1}{c_{44}} .
\end{gathered}
$$

\section{3. Результаты}

Результаты расчета коэффициентов матрицы податливости, экстремальных и средних значений коэффициента Пуассона по формулам (2-4), а также показателей анизотропии кристаллов $\mathrm{Ti}-\mathrm{Ni}$ на основе данных по упругим постоянным $c_{i j}$ представлены в Табл. 1.

Как видно минимальный коэффициент Пуассона кристаллов $\mathrm{TiNi}$ равен -0.25, стандартное отклонение SD 0.16 (для данных из [6]). То есть сплавы на основе Ti-Ni можно рассматривать как частичные ауксетики материалы с отрицательным коэффициентом Пуассона $\mu<0$ (в определенных направлениях). Максимальное значение $\mu$ достигло 1.10 (SD 0.20) (также для данных из [6]), что больше теоретического предела, как известно, равного 0.5 для поликристаллов. Среднее значение $\mu_{\min }$ составило -0.02 , для величин $\mu_{\max } 0.83$ и 0.41 у параметра $<\mu>$; медиана же $--0.005,0.855$, и 0.412 , соответственно (Рис. 1). Таким образом статистическая вариабельность данных по $<\mu>$ SD 0.02 была гораздо меньше, чем у $\mu_{\min }$ и $\mu_{\max }-$ примерно в 8 и 10 раз, соответственно.

Коэффициент упругой анизотропии (по Зинеру) $A=2 c_{44} /\left(c_{11}-c_{12}\right)$ изменялся от 1.37 до 4.58 со средним значением $A=2.97$ (SD 1.04). Отметим, что максимальное значение $A$ соответствовало крайним по величине экстремальным значениям $\mu$ из Табл. 1, а минимальное, наоборот, наиболее близким (Рис. 2). По мере роста $A$ значения $\mu_{\min }$ и $\mu_{\max }$ монотонно дивергировали. При этом полученная на Рис. 3 картина данных совпала с общим видом кривых $\mu(A)$ для кубических кристаллов (Рис. 3) [26], что несколько неожиданно и требует в последующих исследованиях осмысления, поскольку

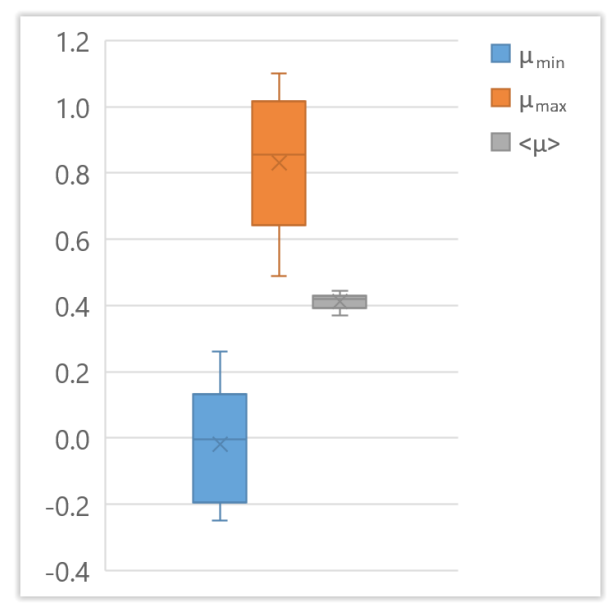

Pис. 1. (Color online) Анизотропия коэффициента Пуассона кристаллов $\mathrm{Ti}-\mathrm{Ni}$.

Fig. 1. (Color online) Anisotropy of the Poisson's ratio of Ti-Ni crystals.

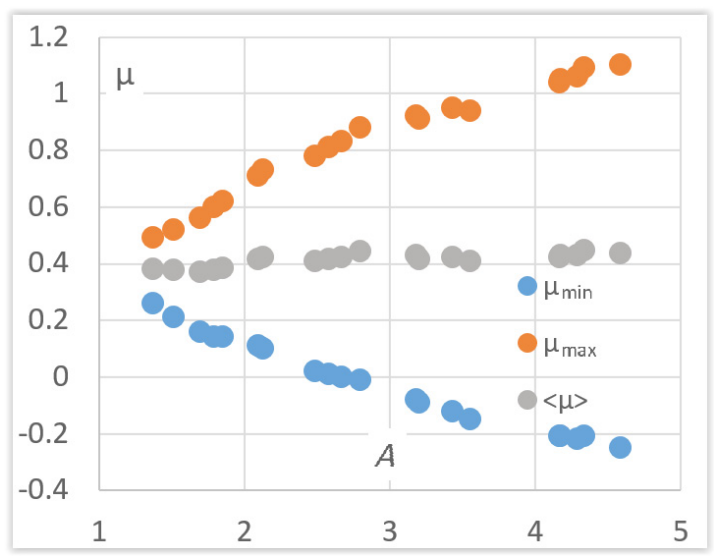

Pис. 2. (Color online) Экстремальные и средние значения коэффициента Пуассона кристаллов $\mathrm{Ti}-\mathrm{Ni}$ в зависимости от коэффициента анизотропии $A$.

Fig. 2. (Color online) Extreme and average values of the Poisson's ratio of Ti-Ni crystals depending on the anisotropy coefficient $A$.

далеко не все кубические кристаллы испытывают мартенситные или какие-либо другие фазовые превращения в широком интервале температур.

Отметим еще одно важное сходство Рис. 2 и 3 . По данным Paszkiewicz T. и Wolski S., 2007 [27] все кубические материалы с экспериментально определенными $A>3$ демонстрируют отрицательные значения коэффициента Пуассона. Это не противоречит ни Рис. 2, ни Рис. 3 (а также данным Табл. 1).

Установленная ярко выраженная анизотропия коэффициента Пуассона кристаллов $\mathrm{Ti}-\mathrm{Ni}$, представленная с помощью Рис. 1, 2 и Табл. 1, привела нас к мысли определить упругую анизотропию кристаллов через различия в маргинальных (терминальных) значениях $\mu$. На наш взгляд было бы логично определить упругую анизотропию кристаллов в значениях отношения $A_{\mu}$, где

$$
A_{\mu}=\frac{\left|\mu_{\max }-\mu_{\min }\right|}{<\mu>} .
$$

Численные значения фактора $A_{\mu}$ включены в Табл. 1 . Видно, что они несколько отличаются от соответст- 
Табл. 1. (Color online) Коэффициенты податливости, экстремальные и средние значения коэффициента Пуассона и коэффициенты анизотропии кристаллов Ti-Ni.

Table 1. (Color online) Compliance coefficients, extreme and average values of the Poisson's ratio and anisotropy coefficients of Ti-Ni crystals.

\begin{tabular}{|c|c|c|c|c|c|c|c|c|c|c|c|}
\hline$c_{11}$ & $c_{12}$ & $c_{44}$ & $s_{11}$ & $s_{12}$ & $s_{44}$ & $A$ & $\mu_{\min }$ & $\mu_{\max }$ & $\langle\mu>$ & $A_{\mu}$ & Ссылки / Ref. \\
\hline 176.5 & 156.1 & 46.8 & 0.033 & -0.015 & 0.0213 & 4.588235 & -0.25 & 1.1 & 0.435 & 3.103448 & {$[6]$} \\
\hline 179.5 & 156.4 & 49.5 & 0.029 & -0.013 & 0.0202 & 4.285714 & -0.22 & 1.06 & 0.429 & 2.983683 & {$[6]$} \\
\hline 183 & 146 & 46 & 0.018 & -0.008 & 0.0217 & 2.486486 & 0.02 & 0.78 & 0.41 & 1.853659 & {$[8]$} \\
\hline 178.2 & 147.6 & 49 & 0.022 & -0.01 & 0.0204 & 3.202614 & -0.09 & 0.91 & 0.415 & 2.409639 & {$[9]$} \\
\hline 162.4 & 129.2 & 34.8 & 0.020 & -0.009 & 0.0287 & 2.096386 & 0.11 & 0.71 & 0.416 & 1.442308 & {$[10]$} \\
\hline 175 & 152 & 48 & 0.029 & -0.013 & 0.0208 & 4.173913 & -0.21 & 1.05 & 0.429 & 2.937063 & {$[11]$} \\
\hline 164.5 & 133.5 & 33 & 0.022 & -0.009 & 0.0303 & 2.129032 & 0.1 & 0.73 & 0.422 & 1.492891 & {$[12]$} \\
\hline 209 & 183 & 36.4 & 0.026 & -0.012 & 0.0274 & 2.8 & -0.01 & 0.88 & 0.443 & 2.009029 & {$[13]$} \\
\hline 180 & 150 & 40 & 0.022 & -0.01 & 0.025 & 2.666667 & 0 & 0.83 & 0.424 & 1.957547 & {$[14]$} \\
\hline 190 & 136 & 50 & 0.013 & -0.005 & 0.02 & 1.851852 & 0.14 & 0.62 & 0.385 & 1.246753 & {$[15]$} \\
\hline 170.5 & 145.5 & 39.8 & 0.027 & -0.012 & 0.0251 & 3.184 & -0.08 & 0.92 & 0.428 & 2.336449 & {$[16]$} \\
\hline 169 & 138 & 40 & 0.022 & -0.01 & 0.025 & 2.580645 & 0.01 & 0.81 & 0.417 & 1.918465 & {$[17]$} \\
\hline 180 & 162 & 39 & 0.037 & -0.017 & 0.0256 & 4.333333 & -0.21 & 1.09 & 0.445 & 2.921348 & {$[18]$} \\
\hline 204.6 & 135.3 & 47.5 & 0.010 & -0.004 & 0.021 & 1.370851 & 0.26 & 0.49 & 0.379 & 0.60686 & {$[19]$} \\
\hline 200 & 134 & 56 & 0.010 & -0.004 & 0.0178 & 1.69697 & 0.16 & 0.56 & 0.37 & 1.081081 & {$[20]$} \\
\hline 168 & 144 & 50 & 0.028 & -0.013 & 0.02 & 4.166667 & -0.21 & 1.04 & 0.422 & 2.962085 & {$[21]$} \\
\hline 188 & 131 & 51 & 0.012 & -0.005 & 0.0196 & 1.789474 & 0.14 & 0.6 & 0.378 & 1.216931 & {$[22]$} \\
\hline 184 & 156 & 48 & 0.024 & -0.011 & 0.0208 & 3.428571 & -0.12 & 0.95 & 0.421 & 2.541568 & {$[23]$} \\
\hline 218 & 178 & 71 & 0.017 & -0.007 & 0.014 & 3.55 & -0.15 & 0.94 & 0.41 & 2.658537 & {$[24]$} \\
\hline 204 & 134 & 53 & 0.010 & -0.004 & 0.0187 & 1.514286 & 0.21 & 0.52 & 0.376 & 0.824468 & {$[25]$} \\
\hline
\end{tabular}

*Курсивом отмечены данные, взятые из ссылок, красным - отрицательные значения $\mu_{\min }$.

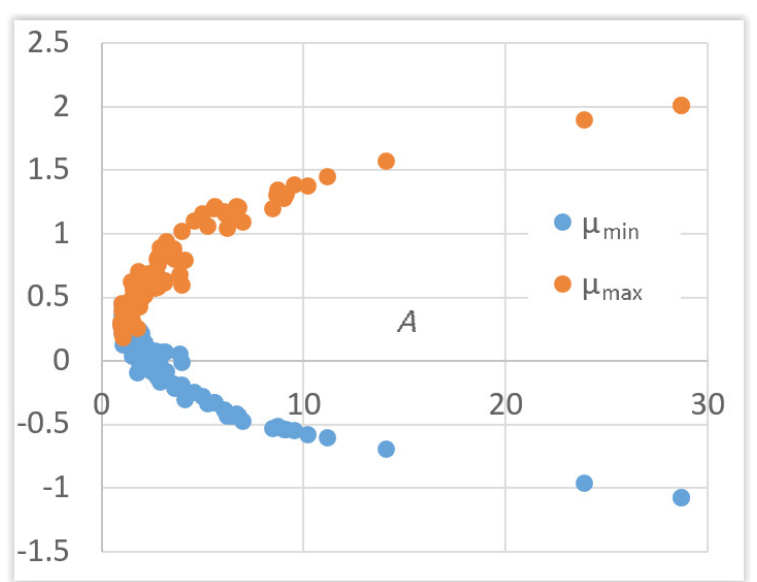

Pис. 3. (Color online) Коэффициент Пуассона кубических кристаллов и его зависимость от коэффициента анизотропии $A$ [26]. Ось абсцисс отделяет области на графике нормального и ауксетичного поведения материалов.

Fig. 3. (Color online) Poisson's Ratio of cubic crystals as a function of the anisotropy coefficient $A$ [26]. Abscissa separates the areas of normal and auxetic behavior.

вующих значений $A$ (Рис. 4), но коррелируют с ними (коэффициент корреляции 0.9871, Excel 2016). Рост $A$ вызывает линейный рост $A_{\mu}$ по закону $A_{\mu}=0.7475 A-0.1386$ (достоверность аппроксимации $R^{2}=0.9744$ ). При этом, если ввести некий приведенный эмпирический фактор $A_{\mu}{ }^{*}=1.33779 A_{\mu}+0.18542$, тогда и вовсе $A_{\mu}^{*}=A$ с высокой степенью точности.

Из Рис. 5 следует, что величина фактора $A_{\mu}$ несколько ниже, чем $A$ : минимальное значение 0.61 , максимальное значение 3.10 , среднее значение $A_{\mu} 2.03$, медиана 1.91 . Размах $A_{\mu}$ также немного меньше, чем у $A$ (SD 0.79).

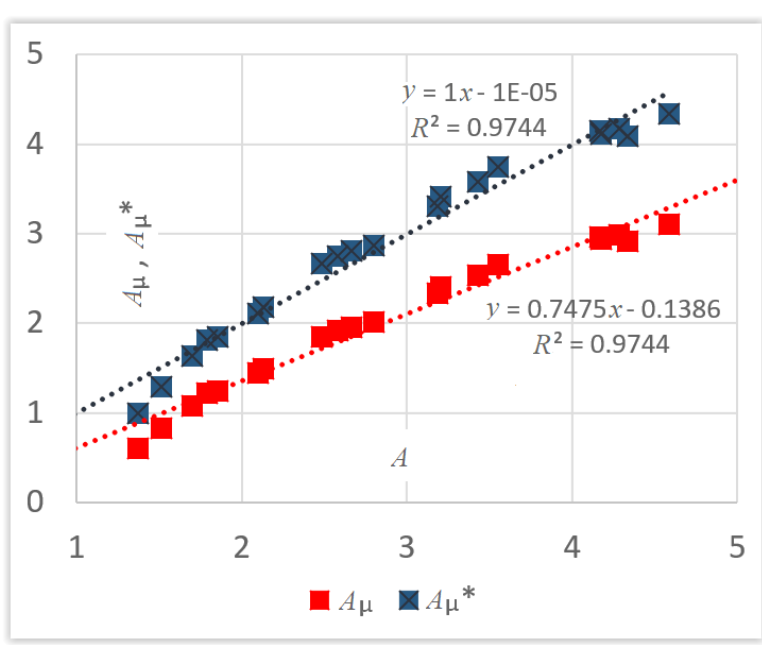

Pис. 4. (Color online) Зависимость фактора анизотропии $A_{\mu}$ и приведенного фактора анизотропии $A_{\mu}^{*}$ от коэффициента упругой анизотропии $A$.

Fig. 4. (Color online) Dependence of the anisotropy factor $A_{\mu}$ and the reduced anisotropy factor $A_{\mu}{ }^{*}$ on the elastic anisotropy coefficient $A$.

\section{4. Выводы}

1. Значения упругих постоянных и коэффициента упругой анизотропии кристаллов $\mathrm{Ti}-\mathrm{Ni}$ с памятью формы весьма вариабельны. Вероятно, это связано c «размягчением» кристаллической решетки вблизи мартенситных превращений (особенно $c_{44}$ накануне B2-R перехода, вследствие чего коэффициент анизотропии $A=c_{44} / C^{\prime}$ может критически изменяться).

2. Минимальный коэффициент Пуассона кристаллов $\mathrm{Ti}-\mathrm{Ni}$ равен -0.25 (SD 0.16). Максимальное значение $\mu$ 


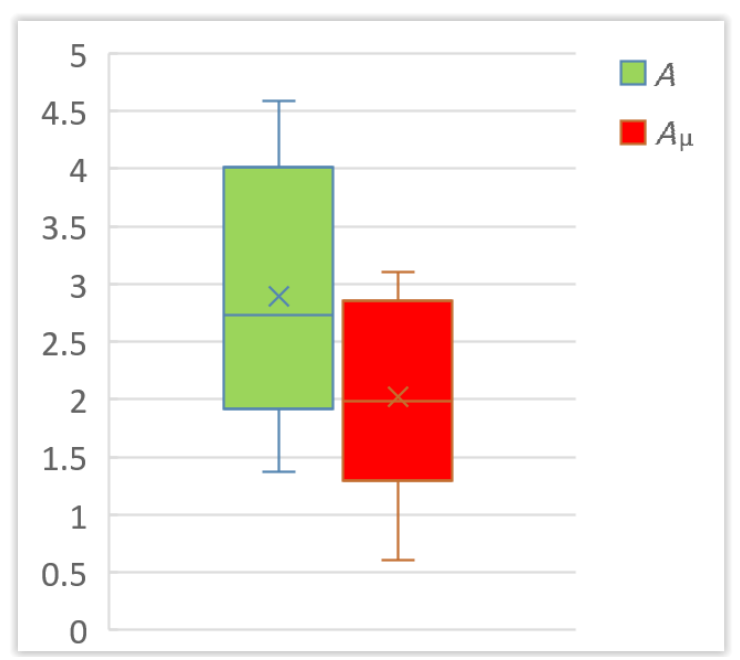

Puc. 5. (Color online) Box and whiskers $A$ и $A_{\mu}$ кристаллов Ti-Ni. Fig. 5. (Color online) Box and whiskers $A$ and $A_{\mu}$ of Ti-Ni crystals.

составило 1.10 (SD 0.20), что больше теоретического предела для поликристаллов. Среднее значение $<\mu>-0.41$ (SD 0.02).

3. Сплавы на основе Ti-Ni могут быть рассмотрены как частичные ауксетики - материалы с отрицательным коэффициентом Пуассона в определенных направлениях.

4. Упругая анизотропия кристаллов $\mathrm{Ti}-\mathrm{Ni}$ может быть определена на основе численных различий экстремальных значений $\mu$ как $A_{\mu}=\left|\mu_{\max }-\mu_{\min }\right| /\langle\mu>$. Установлена тесная положительная корреляционная связь между коэффициентами упругой анизотропии $A$ и $A_{\mu}$ (коэффициент корреляции 0.9871).

Дополнительный материал / Supplementary material. Электронная версия статьи содержит дополнительньй материал (Pис. S1), доступный безвозмездно на сайте журнала (lettersonmaterials.com). / The online version of this paper contains supplementary material (Fig. S1) available free of charge at the journal's Web site (lettersonmaterials.com).

\section{Литература/References}

1. C. Zener. Elasticity and Anelasticity of Metals. University of Chicago, Chicago (1948).

2. V.N. Khachin, S.A. Muslov, V.G. Pushin, Yu. I. Chumlyakov. Reports of the USSR Academy of Sciences. 295 (3), 606 (1987). (in Russian) [В.Н. Хачин, С. А. Муслов, В. Г. Пушин, Ю.И. Чумляков. Доклады AH CCCP. 295 (3), 606 (1987).]

3. A.I. Epishin, D.S. Lisovenko. Journal of technical physics. 86 (10), 74 (2016). (in Russian) [А. И. Епишин, Д.С. Лисовенко. Журнал технической физики. 86 (10), 74 (2016).]

4. D. A. Konyok, K. V. Voitsekhovski, Yu. M. Pleskachevsky, S.V. Shilko. Mechanics of Composite Materials and Structures (Moscow). 10 (1), 35 (2004). (in Russian) [Д. А. Конёк, К. В. Войцеховски, Ю.М.Плескачевский,
С.В. Шилько. Механика композитных материалов и конструкций (Москва). 10 (1), 35 (2004).]

5. Negative Poisson's ratios in metal nanoplates. (in Russian) [Отрицательные коэффициенты Пуассона в металлических нанопластинках.] Electronic resource

6. X. F. Wang, T. E. Jones, W. Li, Y. C. Zhou. Phys. Rev. B. 85, 134108 (2012). Crossref

7. K. W. Wojciechowski. Computational methods in science and technology. 11 (1), 73 (2005). Crossref

8. N. Hatcher, O. Y. Kontsevoi, A. J. Freeman. Phys. Rev. B. 80, 144203 (2009). Crossref

9. G. Bihlmayer, R. Eibler, A. Neckel. Phys. Rev. B. 50, 13113 (1994). Crossref

10. O. Mercier, K. N. Melton, G. Gremaud, J. Hagi. J. Appl. Phys. 51, 1833 (1980). Crossref

11. R. Gaillac, F.-X. Coudert. Elastic tensor analysis. Website

12. S. A. Muslov. Premartensitic states in single crystals of TiNi-TiFe and TiNi-TiCu alloys. Thesis for the degree of candidate of physical and mathematical. Tomsk State University, Tomsk (1987) 166 p. (in Russian) [C.А. Муслов. Предмартенситные состояния в монокристаллах сплавов $\mathrm{TiNi}-\mathrm{TiFe}$ и TiNi-TiCu: дисс. канд. ф.-м. наук. Томск (1987) 166 с.]

13. X. Ren, K. Taniwaki, K. Otsuka, T. Suzuki, K. Tanaka, Yu. I. Chumlyakov. Philos.Mag. A. 79(1), 31 (1999). Crossref

14. X. Ren, N. Miura, J. Zhang, K. Otsuka, K. Tanaka, M. Koiwa, T. Suzuki, Yu. I. Chumlyakov. Mat. Sci. Eng. A. 312, 196 (2001). Crossref

15. P. Šesták, M. Černý, J. Pokluda. Elastic Constants of Austenitic and Martensitic Phases of NiTi Shape Memory Alloy. In: Recent Advances in Mechatronics (ed. by T. Brezina, R. Jablonski). Springer, Berlin, Heidelberg (2010) pp. 1-6.

16. Z.-Y. Zeng et.al. Physica B. 405, 3665 (2010). Crossref

17. M. F.-X. Wagner, W. Windl. Acta Mater. 56, 6232 (2008). Crossref

18. X. Huang, C. Bungaro, V. Godlevsky, K. M. Rabe. Phys. Rev. B. 65, 014108 (2001). Crossref

19. W.S. Lai, B.X. Liu. J. Phys.: Condens. Matter. 12, L53 (2000).

20. J.-M. Lu, Q.-M. Hu, R. Yang. Acta Mater. 56, 4913 (2008). Crossref

21. Y.Y. Ye, C. T. Chan, K.M. Ho. Phys. Rev. B. 56, 3678 (1997). Crossref

22. F. Yu, Y.Liu. Computation. 7(4), 57 (2019). $\underline{\text { Crossref }}$

23. J. B. Haskins, J. W. Lawson. J. Appl. Phys. 121, 205103 (2017). Crossref

24. D. Y. Cheng, S. J. Zhao, S. Q. Wang, H. Q. Ye. Philos. Mag. A. 81, 1625 (2001). Crossref

25. Q.M. Hu, R. Yang, J.M. Lu, L. Wang, B. Johansson, L. Vitos. Phys. Rev. B. 76, 224201 (2007). Crossref

26. Z.A. D. Lethbridge, R.I. Walton, A.S. H. Marmier, C. W. Smith, K.E. Evans. Acta Materialia. 58 (19), 6444 (2010). $\underline{\text { Crossref }}$

27. T. Paszkiewicz, S. Wolski. Phys Status Solidi B - Basic Solid State Phys. 244 (3), 966 (2007). Crossref 